\title{
Idempotent Subreducts of Semimodules over Commutative Semirings
}

\author{
DAVID STANOVsKÝ (*)
}

\begin{abstract}
A short proof of the characterization of idempotent subreducts of semimodules over commutative semirings is presented. It says that an idempotent algebra embeds into a semimodule over a commutative semiring, if and only if it belongs to the variety of Szendrei modes.
\end{abstract}

\section{Introduction.}

Embedding one class of structures into a better understood one usually brings some new knowledge about the former class. We will focus on embeddings of algebras into reducts of semimodules over commutative semirings; hence we obtain linear representations for operations of the algebras.

Modes are idempotent algebras where every pair of operations commute with one another [10]. Indeed, idempotent subreducts of semimodules over commutative semirings are modes and it had been an open problem [10] whether the converse statement is true. Quite recently, N. Dojer observed that such modes satisfy the so-called Szendrei identities (they appeared in the paper [16] by Ágnes Szendrei) and Michal Stronkowski found a syntactical proof that these identities do not follow from the axioms of modes [14]. Thus there exist modes that are not idempotent subreducts of semimodules over commutative semirings; in fact, we present a simple example of such a mode in Example 2.

(*) Indirizzo dell'A.: Charles University in Prague, KA MFF UK, Sokolovská 83, 18675 Praha 8, Czech Republic.

E-mail: stanovsk@karlin.mff.cuni.cz

This work is a part of the research project MSM 0021620839 financed by MŠMT ČR. The author was partly supported by the GAČR grant \#201/05/0002 and by the INTAS grant \#03-51-4110. 
Shortly after that, Stronkowski also proved that Szendrei modes are embeddable [15] and thus obtained the following characterization:

Theorem 1 (M. Stronkowski [15]). An idempotent algebra is a subreduct of a semimodule over a commutative semiring if and only if it is a Szendrei mode.

The aim of the present paper is to provide a short proof of Theorem 1 .

Actually, M. Stronkowski considered a more general situation: He proved the embedding theorem for (not necessarily idempotent) entropic algebras with onto operations. (Theorem 1 is an obvious corollary of this result.) The payoff for greater generality is much greater complexity of his proof; it does not simplify straightforwardly if idempotency is assumed. However, in the idempotent case, one can use several technical tricks developed by Á. Szendrei in [16], which make our proof rather short and transparent. Since modes have interested a number of mathematicians recently (see the monograph [10]), I think presenting a short proof is worthwhile.

The core of the proof of Theorem 1 is contained in Section 3. In Section 2 , we present auxiliary results on free Szendrei modes, based mostly on the original Szendrei's paper [16]. Some partial results related to Theorem 1 can be found in [4][5][6][11][12][18]; a significant part of the survey [9] was devoted to the problem. Motivated by Example 2, the paper [7] is concerned with a broad class of modes that do not embed into semimodules. Related problems are discussed in the last section.

We quickly recall basic definitions. By a commutative semiring we mean an algebra $\boldsymbol{R}=(R,+, \cdot)$ such that both operations,$+ \cdot$ are commutative and associative and distributive laws hold. A semimodule over a semiring $\boldsymbol{R}$ (or an $\boldsymbol{R}$-semimodule) is a "module without subtraction", it means an algebra $\boldsymbol{M}=(M,+, r \cdot: r \in R)$ such that $(M,+)$ is a commutative semigroup and $r$ are unary operations of multiplication by elements of $\boldsymbol{R}$ satisfying associative and distributive laws. Moreover, the semiring in our construction will be unitary, that is, it contains a unit element 1 which acts on semimodules as identity. Note that in $\boldsymbol{R}$-semimodules, a term $t$ over variables $x_{1}, \ldots, x_{n}$ can always be written (uniquely) as

$$
t=r_{1} \cdot x_{1}+\ldots+r_{n} \cdot x_{n}, \quad \text { for some } r_{1}, \ldots, r_{n} \in R .
$$

An algebra $\boldsymbol{A}$ is called a reduct of an algebra $\boldsymbol{B}$, if all operations of $\boldsymbol{A}$ are term operations of $\boldsymbol{B}$. It is called a subreduct, if it is a subalgebra of a reduct of $\boldsymbol{B}$. (Sometimes we also say that $\boldsymbol{A}$ embeds into $\boldsymbol{B}$.) 
In this paper, we consider algebras over an arbitrary signature $\Sigma$ without constant symbols. An algebra is called idempotent, if each element forms a one-element subalgebra. Equivalently, if the identity

$$
f(x, x, \ldots, x) \approx x
$$

holds for every operation $f$. An algebra is called entropic, if every pair of operations commute with one another. Equivalently, if the identity

$f\left(g\left(x_{11}, \ldots, x_{1 n}\right), \ldots, g\left(x_{m 1}, \ldots, x_{m n}\right)\right) \approx g\left(f\left(x_{11}, \ldots, x_{m 1}\right), \ldots, f\left(x_{1 n}, \ldots, x_{m n}\right)\right)$

holds for all operations $f, g$. Idempotent entropic algebras are called modes. The article [9] and the monograph [10] are good surveys of what is known in the theory of modes.

We say that an $n$-ary operation $f$ satisfies Szendrei identities [14][16], if

$$
\begin{aligned}
f\left(f\left(x_{11}, \ldots, x_{1 n}\right), \ldots,\right. & \left.f\left(x_{n 1}, \ldots, x_{n n}\right)\right) \\
& \approx f\left(f\left(x_{\pi(11)}, \ldots, x_{\pi(1 n)}\right), \ldots, f\left(x_{\pi(n 1)}, \ldots, x_{\pi(n n)}\right)\right)
\end{aligned}
$$

holds for every $\pi$, which is the permutation of the $n^{2}$ indices which fixes all indices except $i j$ and $j i$, and switches these two, for some $1 \leq i, j \leq n$. (So we obtain $\left(\begin{array}{l}n \\ 2\end{array}\right)$ identities.) Modes satisfying all Szendrei identities for every operation are called Szendrei modes. Note that Szendrei identities for an operation $f$ imply that $f$ commutes with itself, hence Szendrei algebras with a single operation are entropic. For a binary operation, there is just one Szendrei identity, and it is equivalent to the entropic identity; many authors call this identity mediality [4].

Example 2. We define a ternary operation $f$ on the set $\{0,1,2\}$ by

$$
f(x, y, z)= \begin{cases}2-z & \text { if } x=y=1 \\ z & \text { otherwise }\end{cases}
$$

So $f\left(f\left(x_{1}, x_{2}, x_{3}\right), f\left(y_{1}, y_{2}, y_{3}\right), f\left(z_{1}, z_{2}, z_{3}\right)\right)$ is equal either $2-z_{3}$, if $x_{3}=y_{3}=1$ and $\left(z_{1}, z_{2}\right) \neq(1,1)$, or $z_{1}=z_{2}=1$ and $\left(x_{3}, y_{3}\right) \neq(1,1)$; or it is equal $z_{3}$ otherwise. Consequently, the algebra $\boldsymbol{A}=(\{0,1,2\}, f)$ is a mode. However,

$f(f(0,0,1), f(0,0,0), f(0,1, z))=z \neq 2-z=f(f(0,0,0), f(0,0,0), f(1,1, z))$

for $z \neq 1$, so $\boldsymbol{A}$ is not a Szendrei mode.

The notation and terminology of universal algebra we use is rather standard and follows the book [8]. We assume the standard representation of free algebras in a variety $\mathcal{V}$ by terms modulo the identities of $\mathcal{V}$. Terms 
are considered as labeled rooted trees. Inner nodes are labeled by operation symbols, leaves by variables. Depth of a symbol/variable is defined as the distance from the root.

\section{Free Szendrei modes.}

Throughout the paper, we fix a signature $\Sigma$ without constants (arity of a symbol $\sigma$ will be denoted $\operatorname{ar} \sigma$ ) and let $\Omega$ denote the set of abstract symbols $\alpha_{\sigma, i}$ for every $\sigma \in \Sigma$ and $i=1, \ldots, \operatorname{ar} \sigma$, i.e.

$$
\Omega=\left\{\alpha_{\sigma, i}: \sigma \in \Sigma, i=1, \ldots, \operatorname{ar} \sigma\right\} .
$$

Let $\boldsymbol{R}_{\Sigma}$ denote the semiring with unit $\mathbb{N}[\Omega] / \theta$ of polynomials with (commutative) variables from $\Omega$ and coefficients from the set of natural numbers $\mathbb{N}$, modulo the congruence $\theta$ generated by all pairs

$$
\left(\alpha_{\sigma, 1}+\ldots+\alpha_{\sigma, n}, 1\right)
$$

for every $n$-ary $\sigma \in \Sigma$. On every $\boldsymbol{R}_{\Sigma}$-semimodule $\boldsymbol{M}$, consider the operations $g_{\sigma}$ defined by

$$
g_{\sigma}\left(a_{1}, \ldots, a_{n}\right)=\alpha_{\sigma, 1} \cdot a_{1}+\ldots+\alpha_{\sigma, n} \cdot a_{n}
$$

for every $n$-ary $\sigma \in \Sigma$. Since $\boldsymbol{R}_{\Sigma}$ is a commutative semiring, the algebra $\left(M, g_{\sigma}: \sigma \in \Sigma\right)$ is a Szendrei mode.

For a set $A$, we will denote

- $\boldsymbol{F}(A)=\left(F(A),+, r: r \in R_{\Sigma}\right)$ the free $\boldsymbol{R}_{\Sigma^{-}}$-semimodule over $A$;

- $\boldsymbol{G}(A)=\left(G(A), g_{\sigma}: \sigma \in \Sigma\right)$ the subalgebra of $\left(F(A), g_{\sigma}: \sigma \in \Sigma\right)$ generated by the set $A$.

Clearly, for $u \in F(A)$, we have $u \in G(A)$, iff there is a $\Sigma$-term $t$ such that $u=t\left(a_{1}, \ldots, a_{n}\right)$ for some $a_{1}, \ldots, a_{n} \in A$.

THEOREM 3. The algebra $\boldsymbol{G}(A)$ is a free Szendrei mode over the set A.

The theorem is an easy consequence of results of Á. Szendrei [16]. We outline its proof in the rest of the section.

A term is called completely expanded, if all variables have equal depth. A completely expanded term is called isosceles, if at each particular depth level, all the nodes at that depth are labeled with the same operation symbol, except possibly the variables at the deepest level. E.g., the term $f(g(x, y), g(y, z))$ is isosceles, while $f(g(x, x), h(x, x))$ is not. 
The address of an occurence of a symbol/variable $\sigma$ of depth $k$ in a term $t$ is the sequence $\left(b_{0}, \ldots, b_{k-1}\right)$ of natural numbers such that the (shortest) path from the root to $\sigma$ uses $b_{i}$-th branch of the tree on $i$-th depth level. The trace of an occurence of a symbol/variable $\sigma$ of depth $k$ in a term $t$ is the sequence $\left(\sigma_{0}, \ldots, \sigma_{k-1}\right)$ of operation symbols such that the $i$-th node on the path from the root to $\sigma$ is labeled by $\sigma_{i}$.

Thus, isosceles terms are precisely those terms, where all occurences of variables have the same trace; it is called the trace of an isosceles term. An identity $t \approx s$ is called isosceles, if both $t, s$ are isosceles terms with the same trace.

LemMa 4 ([16], Lemma 2.2). For every pair of terms $t_{1}, t_{2}$, there are isosceles terms $s_{1}, s_{2}$ with the same trace such that $t_{1} \approx s_{1}$ and $t_{2} \approx s_{2}$ are provable from the idempotent identities. Consequently, every identity is equivalent to an isosceles identity (called an isosceles expansion) relative to the idempotent identities.

Let $\Omega^{*}$ denote the set of all monomials

$$
\prod_{\sigma \in \Sigma} \alpha_{\sigma, 1}^{k_{\sigma, 1}} \cdots \alpha_{\sigma, \operatorname{ar} \sigma}^{k_{\sigma, \mathrm{ar} \sigma}}
$$

(it means that all but finitely many $k_{\sigma, i}$ 's are zeros). For a given trace $\tau$, let $\Omega_{\tau}$ denote the set of all $\omega \in \Omega^{*}$ such that for each $\sigma \in \Sigma$, the sum $k_{\sigma, 1}+\ldots+k_{\sigma, \mathrm{ar} \sigma}$ is equal to the number of occurences of $\sigma$ in the trace $\tau$. Thus $\Omega_{\tau}$ consists of monomials that may appear in an interpretation of an isosceles term of trace $\tau$ in $\boldsymbol{G}(A)$.

Lemma 5. Let $\tau$ be a trace and $p=\sum_{\omega \in \Omega_{\tau}} c_{\omega} \omega, q=\sum_{\omega \in \Omega_{\tau}} d_{\omega} \omega$ two polynomials from $\mathbb{N}[\Omega]$. If they are $\theta$-equivalent, then they are equal.

Proof. We pass the situation into the polynomial ring Z[ $Z$ ] : If $p, q$ are $\theta$-equivalent in $\mathbb{N}[\Omega]$, then they are equivalent also in the congruence generated by all pairs $\left(\alpha_{\sigma, 1}+\cdots+\alpha_{\sigma, a r}, 1\right), \sigma \in \Sigma$, in $Z[\Omega]$, and thus $p-q$ belongs to the ideal $\boldsymbol{I}$ of $\mathbb{Z}[\Omega]$ generated by all polynomials $g_{\sigma}=\alpha_{\sigma, 1}+\cdots+\alpha_{\sigma, \text { ar } \sigma}-1, \sigma \in \Sigma$. We prove that this implies $p=q$ by showing that

(*) I does not contain a non-zero polynomial $f=\sum_{\omega \in \Omega_{\tau}} b_{\omega} \omega$ with $b_{\omega} \in Z$ Z.

Note that the following conditions are equivalent: 
(1) $f \in I$;

(2) there exist polynomials $f_{\sigma} \in \mathbb{Z}[\Omega]$ such that $f=\sum_{\sigma \in \Sigma} f_{\sigma} g_{\sigma}$;

(3) in $f$, substituting $1-\alpha_{\sigma, 2}-\ldots-\alpha_{\sigma, \operatorname{ar} \sigma}$ for every $\alpha_{\sigma, 1}$, yields zero polynomial.

It follows from (3) that $\boldsymbol{I}$ is a prime ideal. We prove ( $)$ by induction on $k=\sum_{\sigma \in \tau} \operatorname{ar} \sigma$. If all symbols in $\tau$ have arity 1 , then $f=b \omega$ and it fails condition (3) unless $b=0$. Otherwise, consider a counterexample $f \in \mathbb{Z}[\Omega]$ of minimal degree. Choose an arbitrary $\sigma$ of arity $>1$. The variable $\alpha_{\sigma, n} \in \Omega$ does not divide $f$ : if it $\operatorname{did}$, we had $f=\alpha_{\sigma, n} \cdot g$, and thus, by primeness, $g \in I$ would be a smaller counterexample. So, substituting 0 for $\alpha_{\sigma, n}$ in $f$ yields a non-zero polynomial, which, as follows from (2), belongs to the respective ideal $\boldsymbol{I}$ in $Z\left[\Omega \backslash\left\{\alpha_{\sigma, n}\right\}\right]$, hence we reduced $k$ by one.

Let $t$ be an isosceles term with trace $\tau$. We say that an occurence of a variable in $t$ has the property $\delta(\omega)$ for an $\omega \in \Omega_{\tau}$, if it can be reached by $k_{\sigma, i}$ choices of $i$-th branch in the nodes labeled by $\sigma$. Finally, for every $\omega \in \Omega_{\tau}$, let $\Delta(\omega, x, t)$ denote the number of occurences of the variable $x$ in $t$ with the property $\delta(\omega)$. E.g., if $t=f(g(x, y), g(y, z))$, then $\Delta\left(\alpha_{f, 1} \alpha_{g, 1}, x, t\right)=$ $=\Delta\left(\alpha_{f, 1} \alpha_{g, 2}, y, t\right)=\Delta\left(\alpha_{f, 2} \alpha_{g, 1}, y, t\right)=\Delta\left(\alpha_{f, 2} \alpha_{g, 2}, z, t\right)=1$. If $t=f(f(x, y), f(y, z))$, then $\Delta\left(\alpha_{f, 1} \alpha_{f, 2}, y, t\right)=2$.

LEMMA 6 ([16], Theorem 2.8). The following statements are equivalent for an isosceles identity $t \approx s$.

(1) $t \approx s$ is provable from entropy and Szendrei identities.

(2) $\Delta(\omega, x, t)=\Delta(\omega, x, s)$ for every variable $x$ that occurs in $t$ or $s$ and every $\omega \in \Omega_{\tau}$.

Proposition 7. The following statements are equivalent for terms $t, s$ over variables $x_{1}, \ldots, x_{m}$.

(1) $t \approx s$ holds in all Szendrei modes.

(2) There is an isosceles expansion $t^{*} \approx s^{*}$ of the identity $t \approx s$ that is provable from entropy and Szendrei identities.

(3) Any isosceles expansion $t^{*} \approx s^{*}$ of the identity $t \approx s$ is provable from entropy and Szendrei identities.

(4) $t\left(a_{1}, \ldots, a_{m}\right)=s\left(a_{1}, \ldots, a_{m}\right)$ holds in the algebra $\boldsymbol{G}\left(a_{1}, \ldots, a_{m}\right)$.

Proof. $\quad(3) \Rightarrow(2) \Rightarrow(1) \Rightarrow(4)$ are trivial. We prove (4) $\Rightarrow(3)$.

Assume the equality $t\left(a_{1}, \ldots, a_{m}\right)=s\left(a_{1}, \ldots, a_{m}\right)$ in $\boldsymbol{G}\left(a_{1}, \ldots, a_{m}\right)$. Then 
also $t^{*}\left(a_{1}, \ldots, a_{m}\right)=s^{*}\left(a_{1}, \ldots, a_{m}\right)$ for any isosceles expansion $t^{*} \approx s^{*}$ of the identity $t \approx s$. Let $\tau$ be its trace. Then

$t^{*}\left(a_{1}, \ldots, a_{m}\right)=\sum_{i=1}^{m}\left(\sum_{\omega \in \Omega_{\tau}} c_{i, \omega} \omega\right) \cdot a_{i}$ and $s^{*}\left(a_{1}, \ldots, a_{m}\right)=\sum_{i=1}^{m}\left(\sum_{\omega \in \Omega_{\tau}} d_{i, \omega} \omega\right) \cdot a_{i}$,

where $c_{i \omega}=\Delta\left(\omega, x_{i}, t^{*}\right)$ and $d_{i \omega}=\Delta\left(\omega, x_{i}, s^{*}\right)$. Hence

$$
\sum_{i=1}^{m}\left(\sum_{\omega \in \Omega_{\tau}} c_{i, \omega} \omega\right) \cdot a_{i}=\sum_{i=1}^{m}\left(\sum_{\omega \in \Omega_{\tau}} d_{i, \omega} \omega\right) \cdot a_{i}
$$

holds in the free $\boldsymbol{R}_{\Sigma}$-semimodule over $a_{1}, \ldots, a_{m}$ and, consequently, the polynomials $\sum_{\omega \in \Omega_{\tau}} c_{i, \omega} \omega$ and $\sum_{\omega \in \Omega_{\tau}} d_{i, \omega} \omega$ are $\theta$-equivalent for every $i$, and so, by Lemma 5, are equal. Particularly, $\Delta\left(\omega, x_{i}, t^{*}\right)=c_{i, \omega}=d_{i, \omega}=\Delta\left(\omega, x_{i}, s^{*}\right)$ for every $\omega$ and $i$ and we can use Lemma 6 .

Theorem 3 follows immediately from Proposition 7.

\section{Proof of Theorem 1 .}

Since subreducts of semimodules over commutative semirings satisfy both entropy and Szendrei identities, one implication of Theorem 1 is clear. In the rest of the section, we prove the converse.

Let $\boldsymbol{A}=\left(A, f_{\sigma}: \sigma \in \Sigma\right)$ be an arbitrary Szendrei mode and let's denote $\pi$ the projection of the free Szendrei mode $\boldsymbol{G}(A)=\left(G(A), g_{\sigma}: \sigma \in \Sigma\right)$ onto the algebra $\boldsymbol{A}$, extending the identity mapping on generators. We define a relation $\rho$ on $F(A)$ consisting of all pairs

$$
\left(w+\omega \cdot b, w+\omega \alpha_{\sigma, 1} \cdot a_{1}+\ldots+\omega \alpha_{\sigma, n} \cdot a_{n}\right)
$$

where $\sigma \in \Sigma$ is an $n$-ary symbol, $w \in F(A), \omega \in \Omega^{*}$ and $a_{1}, \ldots, a_{n}, b \in A$ such that $b=f_{\sigma}\left(a_{1}, \ldots, a_{n}\right)$.

Lemma 8. Let $(u, v) \in \rho$. Then $u \in G(A)$ iff $v \in G(A)$. Moreover, if $u, v \in G(A)$, then $\pi(u)=\pi(v)$.

Proof. Let $u \in G(A)$. Then $u=t\left(a_{1}, \ldots, a_{k}\right)$ for some $k$-ary $\Sigma$-term $t$ and some $a_{1}, \ldots, a_{k} \in A$. Since $(u, v) \in \rho$, we have

$$
u=w+\omega \cdot a_{i}
$$


for certain $1 \leq i \leq k$ and

$$
v=w+\omega \alpha_{\sigma, 1} \cdot b_{1}+\ldots+\omega \alpha_{\sigma, n} \cdot b_{n}
$$

for some $n$-ary $\sigma \in \Sigma, w \in F(A), \omega \in \Omega^{*}$ and $b_{1}, \ldots, b_{n} \in A$ such that $a_{i}=f_{\sigma}\left(b_{1}, \ldots, b_{n}\right)$ in $\boldsymbol{A}$. Let $s$ be the term resulting from $t$ by replacing one of the occurence of $a_{i}$ with $\delta(\omega)$ property with the term $\sigma\left(x_{1}, \ldots, x_{n}\right)$, where $x_{1}, \ldots, x_{n}$ are new variables. Then $v=s\left(a_{1}, \ldots, a_{k}, b_{1}, \ldots, b_{n}\right)$ and thus $v \in G(A)$.

[Example: Let $\boldsymbol{A}=(A, *) \quad$ (thus $\left.\Omega=\left\{\alpha_{*, 1}, \alpha_{*, 2}\right\}\right), \quad t=x * y$, $u=t(a, b)=\alpha_{*, 1} a+\alpha_{*, 2} b$, and $b=c * d$. Then $s(x, y, u, v)=x *(u * v)$, and so $\left.v=s(a, b, c, d)=\alpha_{*, 1} a+\alpha_{*, 2} \alpha_{*, 1} c+\alpha_{*, 2} \alpha_{*, 2} d\right]$.

Now, let $v \in G(A)$. Then $v=s\left(a_{1}, \ldots, a_{k}\right)$ for some $k$-ary $\Sigma$-term $s$ and some $a_{1}, \ldots, a_{k} \in A$. Since $(u, v) \in \rho$, we have

$$
u=w+\omega \cdot b
$$

and

$$
v=w+\omega \alpha_{\sigma, 1} \cdot a_{j_{1}}+\ldots+\omega \alpha_{\sigma, n} \cdot a_{j_{n}}
$$

for some $n$-ary $\sigma \in \Sigma, w \in F(A), \omega \in \Omega^{*}$, certain $1 \leq j_{1}, \ldots, j_{n} \leq k$ and $b \in A$ such that $b=f_{\sigma}\left(a_{j_{1}}, \ldots, a_{j_{n}}\right)$. According to Lemma $4, v=s^{\prime}\left(a_{1}, \ldots, a_{k}\right)$ for an isosceles term $s^{\prime}$, and thus, according to Lemma $6, v=s^{\prime \prime}\left(a_{1}, \ldots, a_{k}\right)$ for an isosceles term $s^{\prime \prime}$, in which the involved occurences of $a_{j_{1}}, \ldots, a_{j_{n}}$ are next each other, i.e., they form a subterm $\sigma\left(a_{j_{1}}, \ldots, a_{j_{n}}\right)$. (Recall that Lemma 6 allows to switch any two occurences with the same $\delta(\omega)$ property.) Now, let $t$ be the term that results from $s^{\prime \prime}$ by replacing the subterm $\sigma\left(a_{j_{1}}, \ldots, a_{j_{n}}\right)$ by a single new variable. Then $u=t\left(a_{1}, \ldots, a_{k}, b\right)$ and thus $u \in G(A)$.

[Example: Let $\boldsymbol{A}=(A, *), \quad s=(x * y) *(u * v), \quad v=s(a, b, c, d)=$ $=\alpha_{*, 1} \alpha_{*, 1} a+\alpha_{*, 1} \alpha_{*, 2} b+\alpha_{*, 2} \alpha_{*, 1} c+\alpha_{*, 2} \alpha_{*, 2} d$, and $e=b * d-$ this perfectly fine constellation, since $\alpha_{*, 1} \alpha_{*, 2}=\alpha_{*, 2} \alpha_{*, 1}$. Then $s^{\prime}=s$ and $s^{\prime \prime}=$ $=(x * u) *(y * v)$, so that $v=s^{\prime \prime}(a, b, c, d)$, and we may define $t(x, y, z, u, w)=$ $=(x * u) * w$. Then $\left.u=t(a, b, c, d, e)=\alpha_{*, 1} \alpha_{*, 1} a+\alpha_{*, 1} \alpha_{*, 2} c+\alpha_{*, 2} e.\right]$

So, as we have seen, if $u, v \in G(A)$ and $(u, v) \in \rho$, then we can write $u=t\left(a_{1}, \ldots, a_{k}\right)$ and $v=s\left(a_{1}, \ldots, a_{k}\right)$ for terms $t, s$ such that $s$ results from $t$ by replacing an occurence of a variable $b$ by the subterm $\sigma\left(b_{1}, \ldots, b_{n}\right)$, for some $b, b_{1}, \ldots, b_{n} \in\left\{a_{1}, \ldots, a_{k}\right\}$ with $b=f_{\sigma}\left(b_{1}, \ldots, b_{n}\right)$ in $\boldsymbol{A}$. Hence, because $\pi$ is a homomorphism identical on $A$, we have $\pi(u)=\pi(v)$.

Let $\bar{\rho}$ be the symmetric transitive closure of $\rho$. Then $\bar{\rho}$ is a congruence of

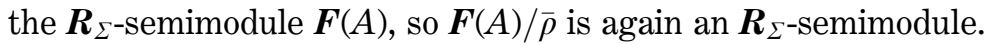


LEMma 9. The Szendrei mode $\boldsymbol{A}$ embeds into the reduct $\left(F(A) / \bar{\rho}, g_{\sigma}: \sigma \in \Sigma\right)$ of the $\boldsymbol{R}_{\Sigma^{-}}$semimodule $\boldsymbol{F}(A) / \bar{\rho}$.

Proof. The embedding is $a \mapsto[a]_{\bar{\rho}}$. This is a homomorphism, because

$$
\begin{aligned}
g_{\sigma}\left(\left[a_{1}\right]_{\bar{\rho}}, \ldots,\left[a_{n}\right]_{\bar{\rho}}\right) & =\alpha_{\sigma, 1} \cdot\left[a_{1}\right]_{\bar{\rho}}+\ldots+\alpha_{\sigma, n} \cdot\left[a_{n}\right]_{\bar{\rho}} \\
& =\left[\alpha_{\sigma, 1} \cdot a_{1}+\ldots+\alpha_{\sigma, n} \cdot a_{n}\right]_{\bar{\rho}}=\left[f_{\sigma}\left(a_{1}, \ldots, a_{n}\right)\right]_{\bar{\rho}} .
\end{aligned}
$$

(The first equality is the definition of $g_{\sigma}$, the last follows from the definition of $\rho$.) So it remains to prove that the mapping is injective. Assume $[a]_{\bar{\rho}}=[b]_{\bar{\rho}}$ for some $a, b \in A$, it means $(a, b) \in \bar{\rho}$. Hence there is a chain $a=u_{0}, u_{1}, \ldots, u_{n-1}, u_{n}=b$ such that $\left(u_{i}, u_{i+1}\right) \in \rho \cup \rho^{-1}$. It follows from Lemma 8 that $u_{0}, \ldots, u_{n} \in G(A)$ and thus that $\pi\left(u_{0}\right)=\pi\left(u_{1}\right)=\ldots=\pi\left(u_{n}\right)$. However, $\pi(a)=\pi(b)$ iff $a=b$, because $\pi$ is the identity on $A$.

This ultimately proves Theorem 1 .

\section{Concluding remarks.}

Two similar types of representation appear in the literature:

- Quasi-(semi)linear algebras are subreducts of (semi)modules; their operations can be expressed as (semi)module terms, i.e. $r_{1} \cdot x_{1}+\ldots+r_{n} \cdot x_{n}$.

- Quasi-(semi)affine algebras are subreducts of (semi)modules with additional constants pointing to every element;

their operations can be expressed as (semi)module polynomials, i.e. $c+r_{1} \cdot x_{1}+\ldots+r_{n} \cdot x_{n}$ with a constant $c$.

In this terminology, what we did, is characterizing idempotent quasisemilinear algebras over commutative semirings.

We wish to discuss a couple of related questions. First, why do we consider idempotent subreducts only? One reason is that my original intention was to answer the open problem posed in [10], to characterize modes embeddable into semimodules over commutative semirings. Even when Stronkowski's result appeared, it was still desirable to find a short and transparent proof for the idempotent case. A characterization of not necessarily idempotent subreducts is an open problem.

Regarding semilinear representations over general semirings, the problem is ultimately solved. J. Ježek [3] proved that actually every algebra (without constants) is a subreduct of a semimodule over a semiring. 
And what about semiaffine representations? Since idempotent quasisemiaffine algebras over commutative semirings are also Szendrei modes, we obtain "quasi-semiaffine over c.s. $\Leftrightarrow$ quasi-semilinear over c.s." for idempotent algebras. However, according to Ježek and Kepka [4], there is a (non-idempotent) algebra which is quasi-semiaffine over c.s. but not quasi-semilinear over c.s.

What about subreducts of modules? We don't know any general results about quasi-linear algebras, but there are several papers on quasi-affine algebras. Indeed, they are abelian, in the sense of commutator theory [1]. Not all abelian algebras are quasi-affine, though this is true under various additional assumptions, such as congruence modularity [2]. R. Quackenbush [13] proved that quasi-affine algebras form a quasivariety, axiomatized by a scheme of quasiidentities that could be considered as a "more restrictive abelianess". For more information, see the survey paper [17]. We don't know whether quasi-affine algebras without constants are quasi-linear.

Finally, let's look at representations over commutative rings. Particularly, which modes are embeddable into modules over commutative rings? Chapter 7 of the book [10] is devoted to this problem. For instance, cancellative modes are quasi-linear, and [15] contains a non-idempotent generalization of this statement. However, no characterization is known. Quasi-linear and quasi-affine algebras are abelian. It is not difficult to prove that abelian modes satisfy Szendrei identities. Is it true that all abelian modes are quasi-linear (or quasi-affine) over commutative rings?

Acknowledgments. The author is indebted to Anna Romanowska and Michal Stronkowski from the Warsaw University of Technology for introducing me to the theory of modes, many helpful advices and also for their hospitality during my visits in Warsaw, confinanced by the GAČR grant \#201/05/0002 and by the INTAS grant \#03-51-4110. I am also grateful to the referee for a thorough reading of the paper and pointing out several weak places.

\section{REFERENCES}

[1] R. Freese - R. McKenzie, Commutator theory for congruence modular varieties, London Mathematical Society Lecture Note Series, 125 (Cambridge University Press, 1987).

[2] C. Herrmann, Affine algebras in congruence modular varieties, Acta. Sci. Math., 41 (1979), pp. 119-125. 
[3] J. JEŽEK, Terms and semiterms, Comment. Math. Univ. Carolinae, 20 (1979), pp. 447-460.

[4] J. JEŽEK - T. KEPKA, Medial groupoids, Rozpravy ČSAV 93/2, 1983.

[5] J. JEŽEK - T. KEPKA, Linear equational theories and semimodule representations, Int. J. Algebra Comput., 8 (1998), pp. 599-615.

[6] K. Kearnes, Semilattice modes I: the associated semiring, Algebra Universalis, 34, 2 (1995), pp. 220-272.

[7] A. Kravchenko - A. Pilitowska - A. Romanowska - D. Stanovský, Differential modes, Internat. J. Algebra Comput., 18, 3 (2008), pp. 567-588.

[8] R. McKenzIe - G. McNulty - W. TaYlor, Algebras, Lattices, Varieties, Volume I. Wadsworth \& Brooks/Cole, 1987.

[9] A. Romanowska, Semi-affine modes and modals, Scientiae Mathematicae Japonicae, 61 (2005), pp. 159-194.

[10] A. Romanowska - JDH Smith, Modes. World Scientific, 2002.

[11] A. Romanowska - A. ZamoJska-DzIEnio, Embedding semilattice sums of cancellative modes into semimodules, Contributions to General Algebra, 13 (2001), pp. 295-303.

[12] A. Romanowska - A. Zamojska-Dzienio, Embedding sums of cancellative modes into semimodules, Czech. Math. J., 55, 4 (2005), pp. 975-991.

[13] R. Quackenbush, Quasi-affine algebras, Algebra Universalis, 20 (1985), pp. $318-327$.

[14] M. Stronkowski, On free modes, Comment. Math. Univ. Carolinae, 47, 4 (2006), pp. 561-568.

[15] M. Stronkowski, On embeddings of entropic algebras, $\mathrm{PhD}$ Thesis, Warsaw University of Technology, 2006.

[16] Á. SzENDREI, Identities satisfied by convex linear forms, Algebra Universalis, 12 (1981), pp. 103-122.

[17] Á. Szendrei, Modules in general algebra, in: Contributions to General Algebra, 10 (Proc. Klagenfurt Conf., 1997), Verlag Johannes Heyn, Klagenfurt, 1998; pp. 41-53.

[18] A. Zamojska-Dzienio, Medial modes and rectangular algebras, Comment. Math. Univ. Carolinae, 47, 1 (2006), pp. 21-34.

Manoscritto pervenuto in redazione il 18 maggio 2007. 
